\title{
Exploration of the Effects of Different Blue LED Light Intensities on Flavonoid and Lipid Metabolism in Tea Plants via Transcriptomics and Metabolomics
}

\author{
Pengjie Wang ${ }^{1}$, Sirong Chen ${ }^{1}$, Mengya Gu ${ }^{1}$, Xiaomin Chen ${ }^{1}$, Xuejin Chen ${ }^{1}$, Jiangfan Yang ${ }^{1}$, \\ Feng Zhao ${ }^{2, *(D)}$ and Naixing Ye ${ }^{1, *(D)}$ \\ 1 College of Horticulture, Fujian Agriculture and Forestry University/Key Laboratory of Tea Science in \\ Universities of Fujian Province, Fuzhou 350002, China; 2180311002@fafu.edu.cn (P.W.); \\ 3170304008@fafu.edu.cn (S.C.); 1190311005@fafu.edu.cn (M.G.); 1190311002@fafu.edu.cn (X.C.); \\ 1180311002@fafu.edu.cn (X.C.); 000q25003@fafu.edu.cn (J.Y.) \\ 2 College of Pharmacy, Fujian University of Traditional Chinese Medicine, Fuzhou 350002, China \\ * Correspondence: zhaofeng0591@fjtcm.edu.cn (F.Z.); 000q020063@fafu.edu.cn (N.Y.)
}

Received: 10 June 2020; Accepted: 25 June 2020; Published: 29 June 2020

\begin{abstract}
Blue light extensively regulates multiple physiological processes and secondary metabolism of plants. Although blue light quantity (fluence rate) is important for plant life, few studies have focused on the effects of different blue light intensity on plant secondary metabolism regulation, including tea plants. Here, we performed transcriptomic and metabolomic analyses of young tea shoots (one bud and two leaves) under three levels of supplemental blue light, including low-intensity blue light (LBL, $50 \mu \mathrm{mol} \mathrm{m} \mathrm{m}^{-2}$ ), medium-intensity blue light (MBL, $100 \mu \mathrm{mol} \mathrm{m}^{-2} \mathrm{~s}^{-1}$ ), and high-intensity blue light (HBL, $200 \mu \mathrm{mol} \mathrm{m} \mathrm{m}^{-2} \mathrm{~s}^{-1}$ ). The total number of differentially expressed genes (DEGs) in LBL, MBL and HBL was 1, 7 and 1097, respectively, indicating that high-intensity blue light comprehensively affects the transcription of tea plants. These DEGs were primarily annotated to the pathways of photosynthesis, lipid metabolism and flavonoid synthesis. In addition, the most abundant transcription factor (TF) families in DEGs were bHLH and MYB, which have been shown to be widely involved in the regulation of plant flavonoids. The significantly changed metabolites that we detected contained 15 lipids and 6 flavonoid components. Further weighted gene co-expression network analysis (WGCNA) indicated that CSMYB (TEA001045) may be a hub gene for the regulation of lipid and flavonoid metabolism by blue light. Our results may help to establish a foundation for future research investigating the regulation of woody plants by blue light.
\end{abstract}

Keywords: Camellia sinensis; blue light; transcriptomics; metabolomics; WGCNA

\section{Introduction}

Plants are sessile in nature and therefore must respond appropriately to changing environmental factors to flourish; of these factors, light is one of the most important. Plants perceive light of different wavelengths through a diverse array of photoreceptors, where phytochromes (PHYA-E) absorb far red and red light, the receptor UVR8 absorbs UV-B light, and cryptochromes (CRY1-3) and phototropins (PHOT1-2) absorb blue and UV-A light [1]. Blue light globally regulates multiple processes in plants and plant cells; these processes include photomorphogenesis, photosynthesis, chloroplast accumulation, stomatal opening, leaf development and flowering time [2-6]. Furthermore, some evidence also suggests that blue light is involved in the molecular regulation of secondary metabolites. For instance, blue light regulates the biosynthesis of functional metabolites, such as rutin and catechins, in longan embryogenic calli, while HY5, PIF4 and MYC2 are considered key regulators $[7,8]$. In citrus, the accumulation of carotenoids and the degradation of chlorophyll occur 
under blue light and lead to deeper and faster coloration of the fruit, and these changes have been related to the upregulation of some structural genes in pigment metabolism [9-11]. Blue light also affects the degradation of fatty acids in plant leaves and changes the fatty acid composition of membrane lipids, and $200 \mu \mathrm{mol} \mathrm{m}^{-2} \mathrm{~s}^{-1}$ blue light can maximize the lipid content of Chlorella [12,13]. These studies indicate that blue light has the potential to regulate plant growth and secondary metabolism.

The tea plant Camellia sinensis (L.) O. Kuntze is an economically important perennial evergreen crop that is widely cultivated worldwide [14]. Normally, the young shoots of tea plants can be processed into tea, one of the most popular nonalcoholic beverages in the world. Tea is rich in secondary metabolites, including flavonoids, caffeine, theanine and volatile compounds, which benefit human health $[15,16]$. Due to the regulation of various secondary metabolites by light, there have been multiple studies in tea plants to control the intensity of light by shading to improve or modify the metabolic components of tea leaves [17-20]. Fu et al. [21] first explored the regulatory effect of single-wavelength blue light $(470 \mathrm{~nm})$ and red light $(660 \mathrm{~nm})$ on the formation of volatile compounds in tea plants. Among these types of light, blue light significantly elevated most endogenous volatiles and activated the expression of structural genes related to the formation of tea plant volatiles. A recent study showed that employing high-intensity supplemental blue light for $4 \mathrm{~h}$ during the nighttime can induce the expression of MYBs, CRY2/3, SPAs and HY5, thereby promoting the accumulation of anthocyanins and catechins in tea leaves [22]. Light quality (wavelength) and quantity (fluence rate) are important to plant life [23,24]. However, to the best of our knowledge, no research to date has investigated the relationship between different blue light intensities and the secondary metabolism of tea plants.

In recent decades, light emitting diodes (LEDs) have been developed rapidly as alternative light sources [25]. LEDs have the advantages of light intensity/quality adjustability, energy savings and durability and have been successfully applied to such crops as cucumber [26], pepper [27], banana [28], tomato [29] and grape [30]. In this study, we attempted to investigate the effects of different blue LED light intensities on the gene expression levels and metabolite profiles of tea plant shoots. Furthermore, we evaluated multiple changes in photosynthesis, flavonoid biosynthesis and lipid metabolism pathways to elucidate how different blue light intensities affect growth and secondary metabolism in tea plants.

\section{Results}

\subsection{Overview of the Transcriptional Changes}

To reveal the molecular regulation of tea plants under different blue light intensities, we performed transcriptome sequencing on tea plant shoots (one bud and two leaves) under three blue LED intensities. As shown in Table 1, a total of 42.90-62.33 million raw reads were obtained, and Q20 and Q30 were greater than $97 \%$ and $93 \%$, respectively, indicating the high throughput and quality of the RNA-Seq data. After filtering out the low-quality reads, 42.60-61.88 million clean reads were subjected to further analysis. Among these reads, $91.89-93.26 \%$ of clean reads were mapped to the tea plant genome. The RNA-Seq data sets were deposited in the NCBI SRA database under accession number PRJNA636584. Subsequently, we evaluated the effects of different blue light intensities on global gene transcript abundance. Compared with white light (CK), the global gene transcript abundance decreased as the blue light intensity increased (Figure 1A).

The numbers of differentially expressed genes (DEGs) observed under different blue light intensities are shown in Figure 1B,C. Interestingly, compared to CK, the total number of DEGs in low-intensity blue light (LBL, $50 \mu \mathrm{mol} \mathrm{m}{ }^{-2} \mathrm{~s}^{-1}$ ), medium-intensity blue light (MBL, $100 \mu \mathrm{mol} \mathrm{m} \mathrm{m}^{-2} \mathrm{~s}^{-1}$ ) and high-intensity blue light (HBL, $200 \mu \mathrm{mol} \mathrm{m}^{-2} \mathrm{~s}^{-1}$ ) was 1, 7 and 1097, respectively, indicating that the effect of low-intensity blue light on tea plant shoots was highly limited. To validate the reliability of the RNA-Seq data, 12 DEGs, including 4 randomly selected DEGs, 2 transcription factors, 2 photosynthesis-related DEGs, 2 flavonoid biosynthesis-related DEGs and 2 lipid biosynthesis-related 
DEGs, were selected to detect their expression levels by qRT-PCR. The results showed that the expression profiles detected by qRT-PCR were positively correlated with the RNA-Seq results (Figure 2).

Table 1. Quality of the transcriptome data of each sample.

\begin{tabular}{cccccc}
\hline Sample & Raw Reads & Clean Reads & Total Mapped & Q20 (\%) & Q30 (\%) \\
\hline CK-1 & 55555128 & 55178152 & $50992408(92.41 \%)$ & 98.37 & 94.72 \\
CK-2 & 62332084 & 61884910 & $57198092(92.43 \%)$ & 98.15 & 94.13 \\
CK-3 & 42897998 & 42595356 & $39331322(92.34 \%)$ & 98.04 & 93.85 \\
LBL-1 & 55216836 & 54854554 & $50956186(92.89 \%)$ & 98.12 & 94.02 \\
LBL-2 & 48161620 & 47741022 & $43871489(91.89 \%)$ & 97.97 & 93.71 \\
LBL-3 & 60688032 & 60256324 & $55807398(92.62 \%)$ & 98.29 & 94.48 \\
MBL-1 & 53013332 & 52623504 & $48866086(92.86 \%)$ & 97.99 & 93.71 \\
MBL-2 & 48309310 & 47996004 & $44758708(93.26 \%)$ & 98.26 & 94.39 \\
MBL-3 & 61387750 & 60995982 & $56491831(92.62 \%)$ & 98.23 & 94.35 \\
HBL-1 & 46838196 & 46520542 & $43145309(92.74 \%)$ & 98.27 & 94.44 \\
HBL-2 & 58747398 & 58408158 & $54130109(92.68 \%)$ & 98.27 & 94.44 \\
HBL-3 & 53331584 & 52950364 & $49149589(92.82 \%)$ & 98.06 & 93.89 \\
\hline
\end{tabular}

A

Expression Distribution

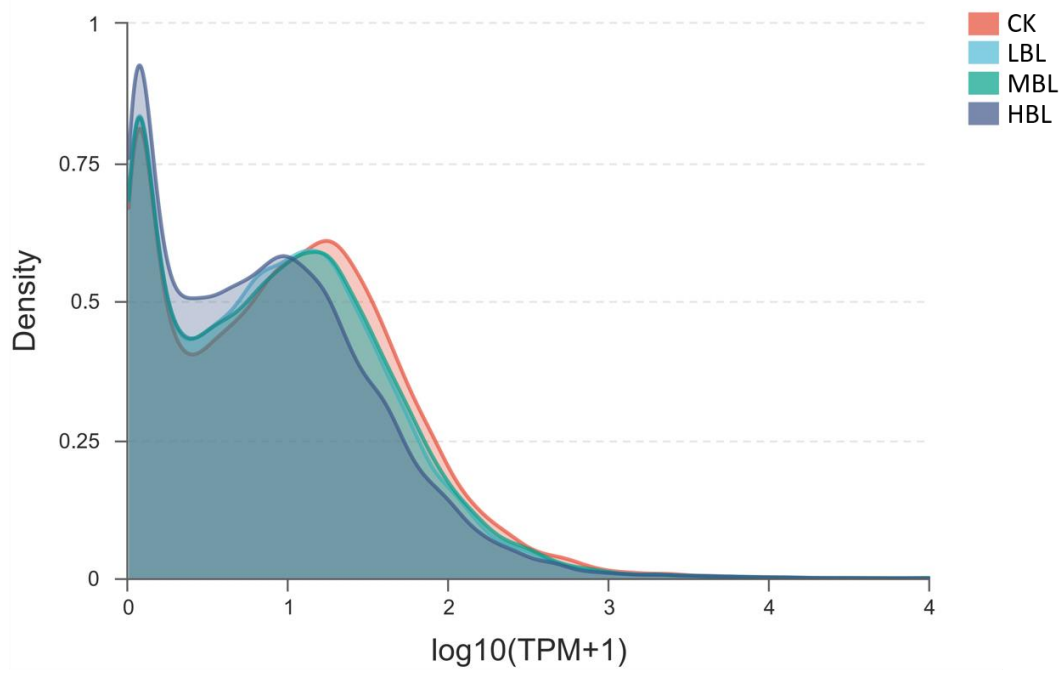

B
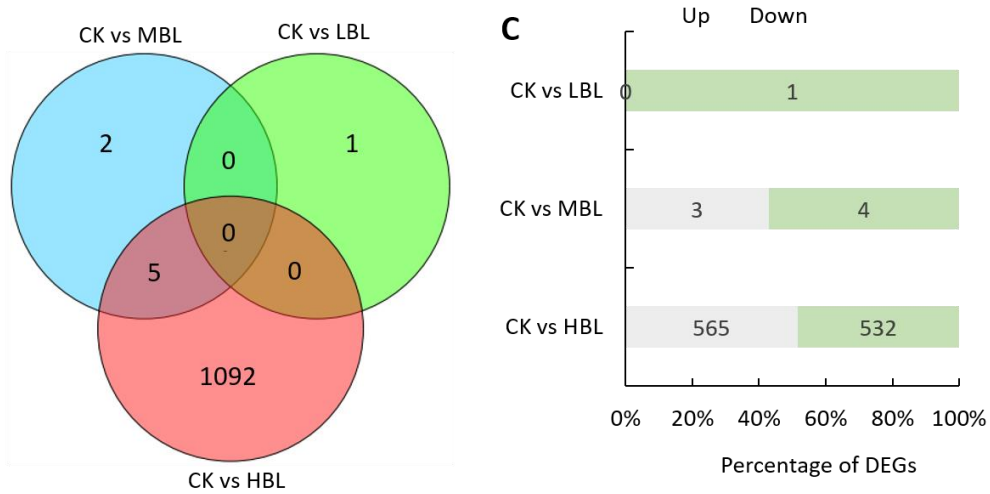

Figure 1. Global gene expression profiling and differentially expressed genes (DEGs) in tea plant shoots under different blue light intensities. (A) Density plot of global gene expression in tea plants under different blue light intensities. (B) Venn diagram of DEGs in tea plants under different blue light intensities. (C) The number of up- and downregulated DEGs in each comparison. 


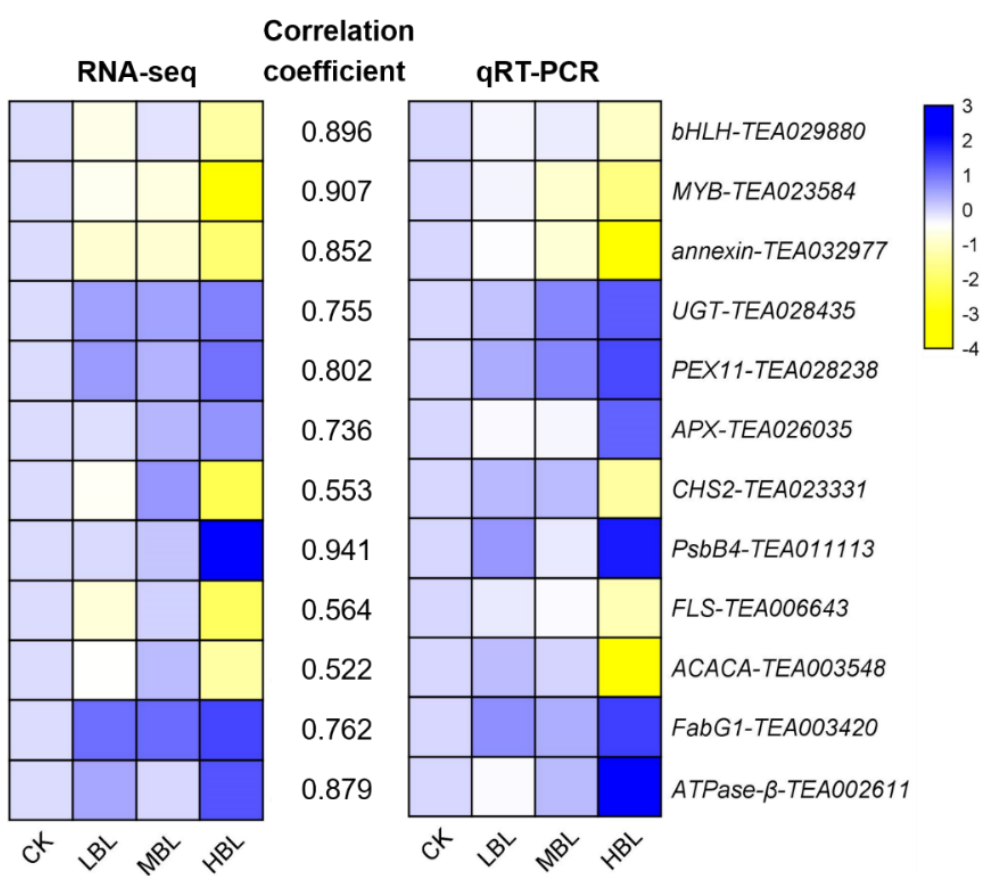

Figure 2. Validation and correlation analysis of 12 selected DEGs. The values between two heatmaps represent the correlation coefficients of qRT-PCR and RNA-seq values from each gene.

\subsection{Annotation of DEGs between $C K$ and $H B L$}

Since DEGs are only enriched between CK and HBL, we further analyzed the global metabolic pathways of the DEGs via iPath3.0 (http://pathways.embl.de) [31]. As shown in Figure 3, most DEGs were annotated to lipid metabolism, flavonoid metabolism, energy metabolism, carbohydrate metabolism and amino acid metabolism. Among these DEGs, photosynthesis and sulfur metabolism in energy metabolism were significantly enriched, indicating that the energy metabolism of tea plant shoots under high-intensity blue light has a significant response, which may affect the synthesis of multiple secondary metabolites.

In Gene Ontology (GO) term enrichment analysis, 57 DEGs were enriched in the 10 most significant GO terms (FDR < 0.05), including beta-glucan metabolic process, carbohydrate catabolic process, polysaccharide catabolic process, photosynthesis, polysaccharide metabolic process, flavonoid biosynthetic process, drug catabolic process, neurotransmitter catabolic process, cellular amino acid catabolic process and carbohydrate metabolic process (Figure 4A and Table S1).

In Kyoto Encyclopedia of Genes and Genomes (KEGG) pathway enrichment analysis, 97 DEGs were annotated in the 10 most significant pathways (FDR < 0.05), including flavone and flavonol biosynthesis, carbon fixation in photosynthetic organisms, photosynthesis, glycine, serine and threonine metabolism, glyoxylate and dicarboxylate metabolism, phenylalanine, tyrosine and tryptophan biosynthesis, monoterpenoid biosynthesis, flavonoid biosynthesis, ubiquinone and other terpenoid-quinone biosynthesis and fatty acid biosynthesis (Figure 4B and Table S2).

Overall, many DEGs that showed distinct expression patterns between CK and HBL were related to photosynthesis and lipid and flavonoid metabolism. Interestingly, the 17 DEGs annotated to photosynthesis were significantly upregulated in HBL (Figure 5), especially four PsaB genes from Photosystem I and one F-type ATPase- $\alpha$ gene, which further suggests that high-intensity blue light regulates energy metabolism in tea plant. 


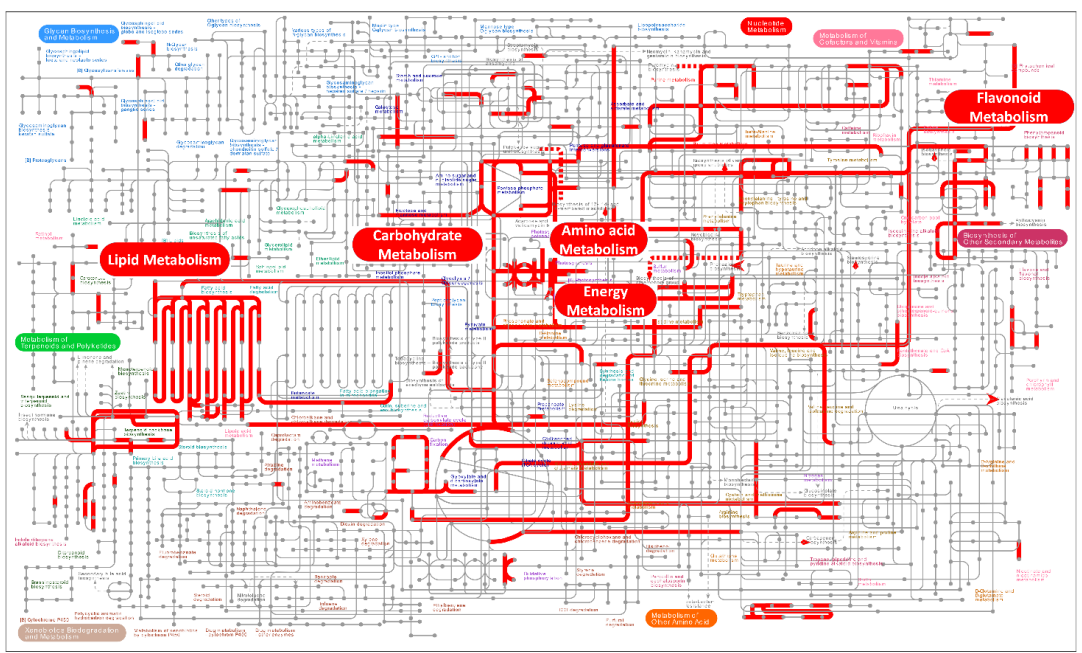

Figure 3. Overview of the metabolic pathways of DEGs between white light (CK) and high-intensity blue light (HBL). The red lines represent pathways annotated by DEGs, and the red boxes represent metabolic pathways that were highly enriched. These metabolic maps were visualized and analyzed by the iPath3.0 online tool (http://pathways.embl.de).
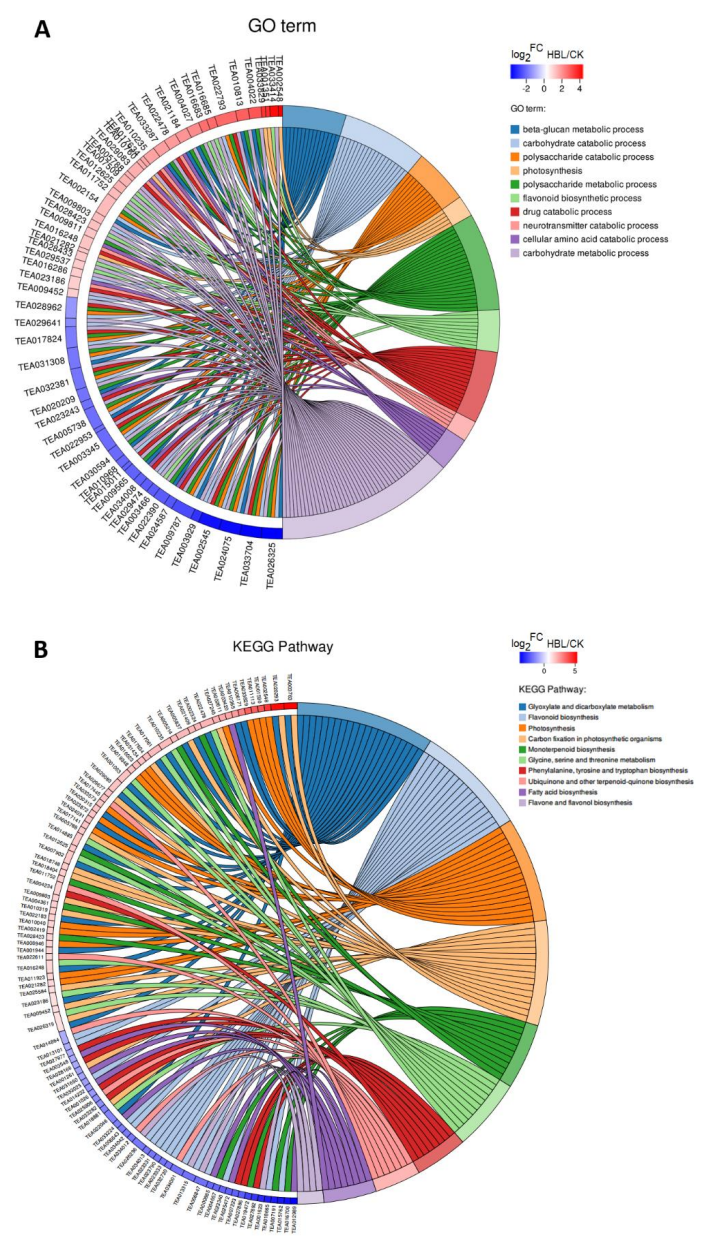

Figure 4. Chord plot of the top 10 Gene Ontology (GO) terms (A) and Kyoto Encyclopedia of Genes and Genomes (KEGG) pathways (B). Chords show a detailed relationship between the $\log _{2}$-fold change $\left(\log _{2} \mathrm{FC}\right)$ of DEGs (left semicircle) and their enriched GO terms or KEGG pathways (right semicircle). 

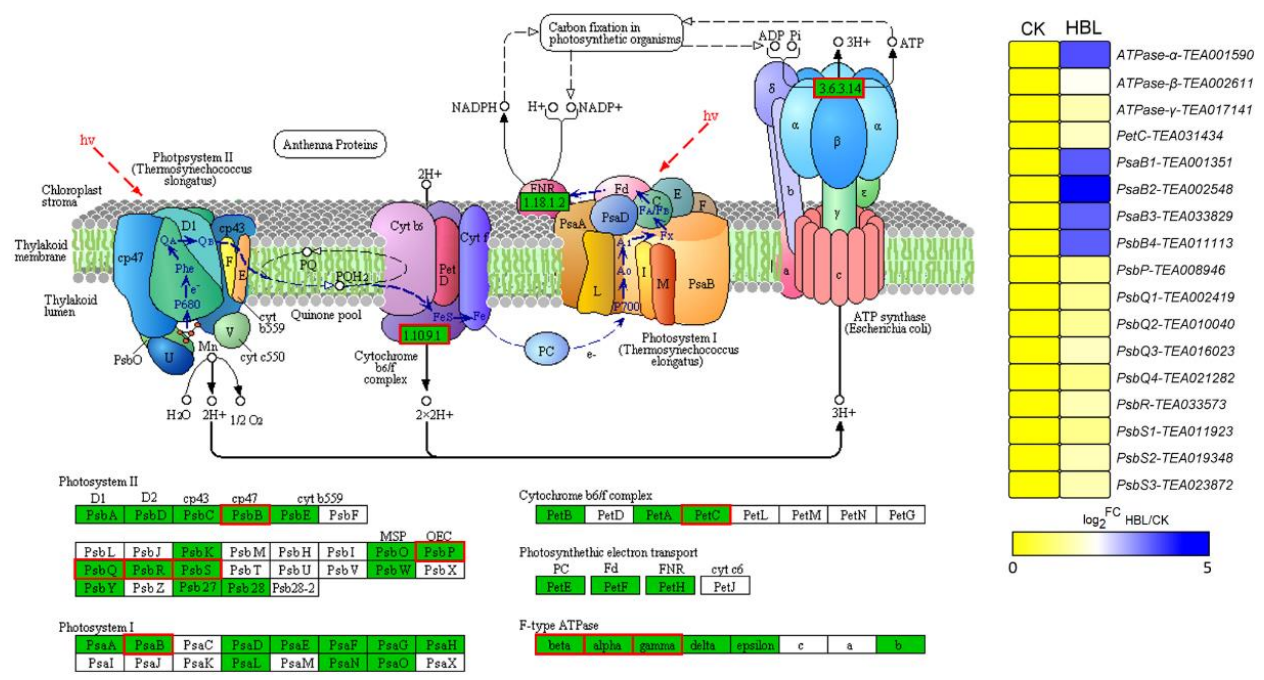

Figure 5. DEGs involved in photosynthesis under high intensity blue light in tea plant shoots. The pathway adapted from KEGG, the green box indicates the background genes of tea plant genome, and the red line indicates the annotated DEGs. The heatmap was generated from the $\log _{2}$-fold change $\left(\log _{2} \mathrm{FC}\right)$ mean value calculated from three replicates of RNA-Seq data.

\subsection{Analysis of DEGs Related to Flavonoid Biosynthesis}

To further explore the effect of high-intensity blue light on structural genes of flavonoid synthesis, the transcriptional abundance of 19 DEGs involved in flavonoid biosynthesis was visualized (Figure 6). The 19 DEGs encode 2 C4H, 2 4CL, 6 CHS, 2 CHI, 1 F3H, 1 DFR, 2 F3'H, 1 FLS and 1 ANS genes. Notably, all structural DEGs were significantly downregulated in HBL to varying degrees, especially CHS (TEA018665) and ANS (TEA015762) coding genes, which were downregulated more than 10-fold, indicating that high-intensity blue light comprehensively inhibited flavonoid metabolism in tea plants.
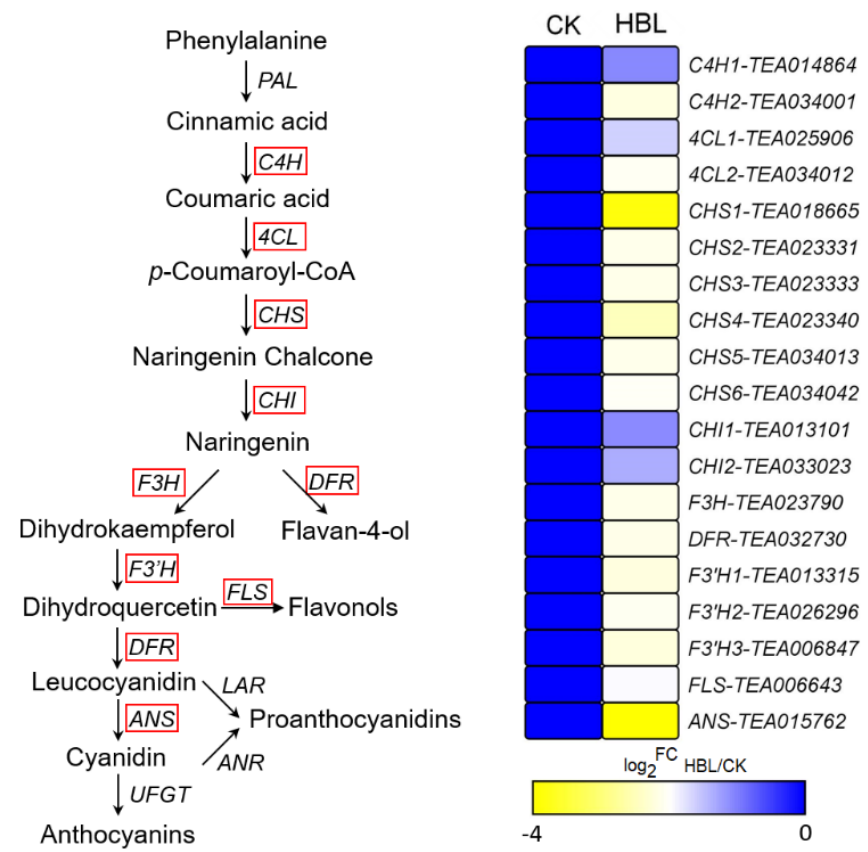

Figure 6. DEGs involved in flavonoid biosynthesis under high intensity blue light in tea plant shoots. The red line indicates the annotated DEGs. The heatmap was generated from the $\log _{2}$-fold change $\left(\log _{2} \mathrm{FC}\right)$ mean value calculated from three replicates of RNA-Seq data. 


\subsection{Analysis of DEGs Related to Lipid Metabolism}

In the fatty acid biosynthesis and degradation pathway of lipid metabolism (Figure 7), only one FabG (TEA003420) was upregulated 4.45-fold in HBL. The other seven structural DEGs, including 1 ACACA, 1 FabF, 2 FabG, 1 FaBI, 1 FATB and 1 ACSL, were generally downregulated in HBL. Overall, fatty acid synthesis in tea plant shoots was inhibited under high-intensity blue light, since the transcriptional abundance of most structural DEGs involved in this pathway was significantly reduced.

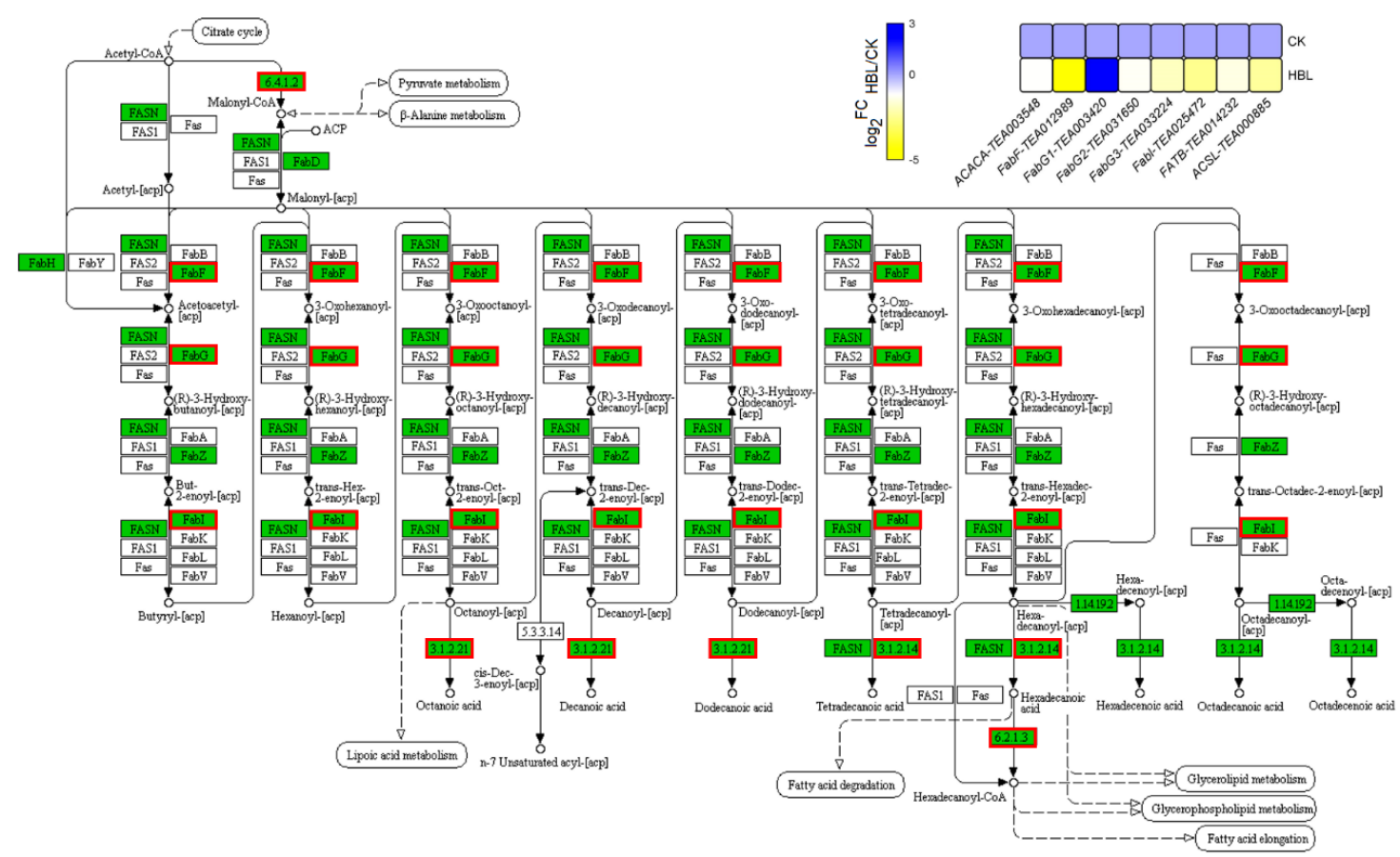

Figure 7. DEGs involved in fatty acid biosynthesis under high-intensity blue light in tea plant shoots. In the pathway adapted from KEGG, the green box indicates the background genes of tea plant genome, and the red line indicates the annotated DEGs. The heatmap was generated from the $\log _{2}$-fold change $\left(\log _{2} \mathrm{FC}\right)$ mean value calculated from three replicates of RNA-Seq data.

\subsection{Analysis of Differentially Expressed Transcription Factors (DETFs)}

Transcription factors (TFs) are vital regulatory factors involved in regulating plant growth and development, while bHLH and MYB TFs have been widely shown to play important roles in the regulation of plant flavonoid accumulation. In our RNA-seq data, 54 differentially expressed transcription factors (DETFs) belonging to $16 \mathrm{TF}$ families were identified between CK and HBL (Figure 8). Among these factors, the most abundant TF families were bHLH $(11,20.37 \%)$ and MYB $(8,14.81 \%)$ followed by AP2/ERF (7, 12.96\%), MYB-related $(5,9.26 \%)$, bZIP $(5,9.26 \%)$ and NAC (4, $7.41 \%)$. Interestingly, most of the bHLH (10/11, 90.90\%) and MYB (6/8, 75.00\%) TFs were significantly downregulated in HBL, which was consistent with the expression trend of related structural DEGs involved in flavonoid biosynthesis. 


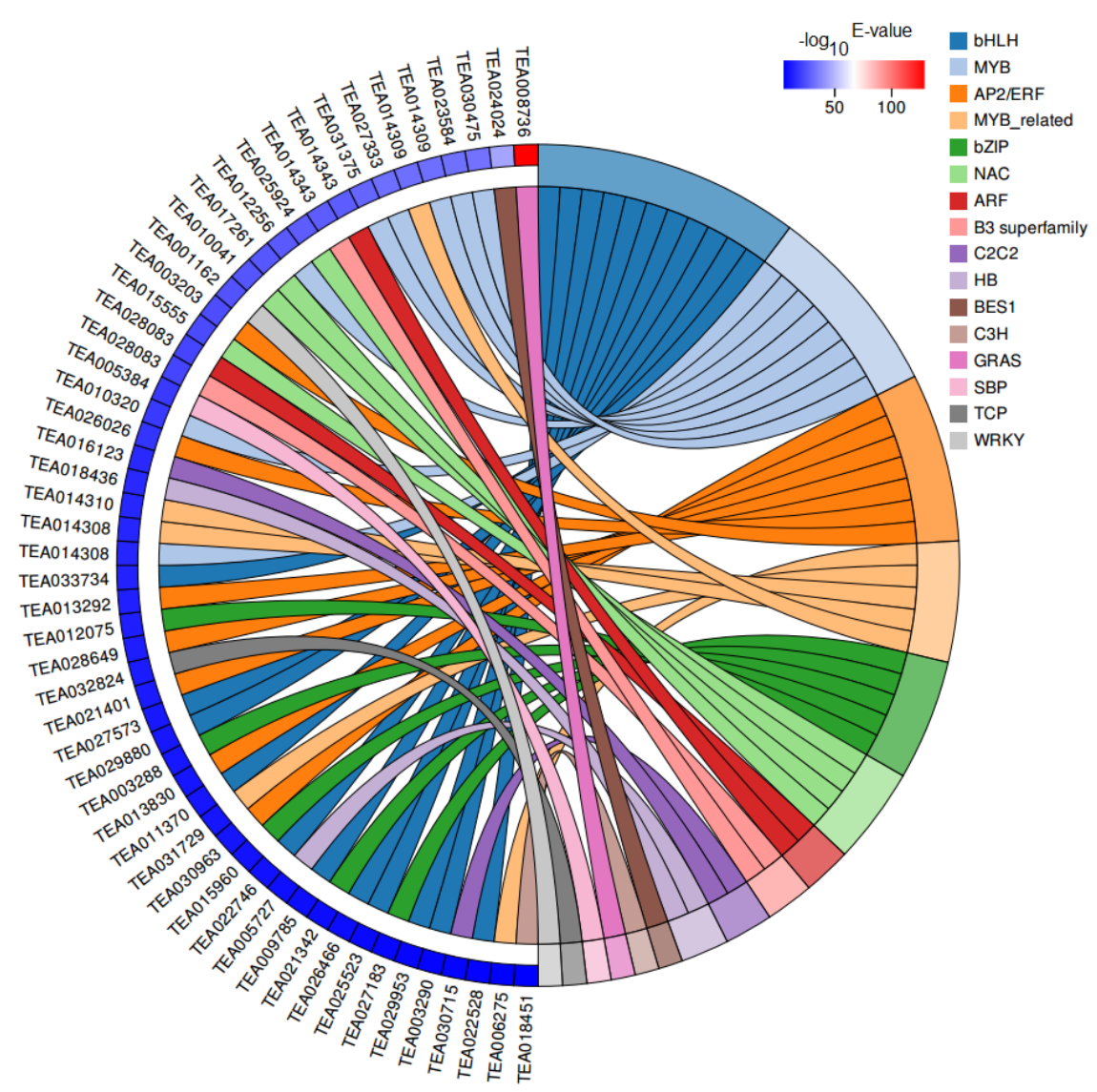

Figure 8. Chord plot of differentially expressed transcription factors (DETFs). Chords show a detailed relationship between the $-\log _{10}$ E-value of DETFs (left semicircle) and their assigned transcription factor families (right semicircle).

\subsection{Metabolite Changes in Response to Different Blue Light Intensities}

A total of 48 significantly changed metabolites (SCMs) were identified in tea plant shoots under different blue light intensities. The number of SCMs upregulated and downregulated in CK versus LBL, CK versus MBL and CK versus HBL were 1 and 0, 7 and 7 and 24 and 9, respectively (Figure 9A). To observe the overall SCM pattern, we further visualized the fold change of SCMs in the clustering heatmap (Figure 9B and Table S3). These SCMs mainly belonged to lipids and lipid-like molecules and flavonoids and a small amount of carbohydrates and carbohydrate conjugates, nucleotides and amino acids and their derivatives. Fifteen lipids and lipid-like molecules can be subdivided into fatty acyls, glycerolipids, glycerophospholipids, prenol lipids, saccharolipids and steroids and steroid derivatives, 7 of which increased in HBL, including 3-oxo-alpha-ionol 9- [apiosyl- (1- > 6) -glucoside], dihydroroseoside, PS (18: 0/20: 3 (8Z, 11Z, 14Z)), PG (16: 1 (9Z)/18: 2 (9Z, 12Z)), hydroxyisonobilin, ichangic acid 17-beta-D-glucopyranoside and 11,13-dihydrotaraxinic acid glucosyl ester, while others, such as DG (16: 0/21: 0/0: 0), CL (a-13: 0/i-24: 0/18: 2 (9Z, 11Z)/i-21: 0) [rac], LysoPC (18: 1 $(11 Z)$ ), cucurbitaxanthin B and vaccinoside decreased. Six flavonoid glycosides were significantly changed under blue light, and the quantity of three flavonoid glycosides was significantly elevated, including 3',5,6-trihydroxy-3,4',7,8-tetramethoxyflavone 3-glucoside, galangin 3- [galactosyl- (1- > 4) -rhamnoside] and neocarthamin, while the level of quercetin 3- (2-caffeoylsophoroside) 7-glucoside, quercetin 3- (4 "-acetylrhamnoside) 7-rhamnoside and spinacetin 3- (2 "-feruloylgentiobioside) was decreased. The metabolite data further showed the effect of blue light on lipid and flavonoid metabolism in tea plants, and as the intensity of blue light increased, the changes in metabolites intensified, which was consistent with the gene expression changes observed by RNA-Seq. 


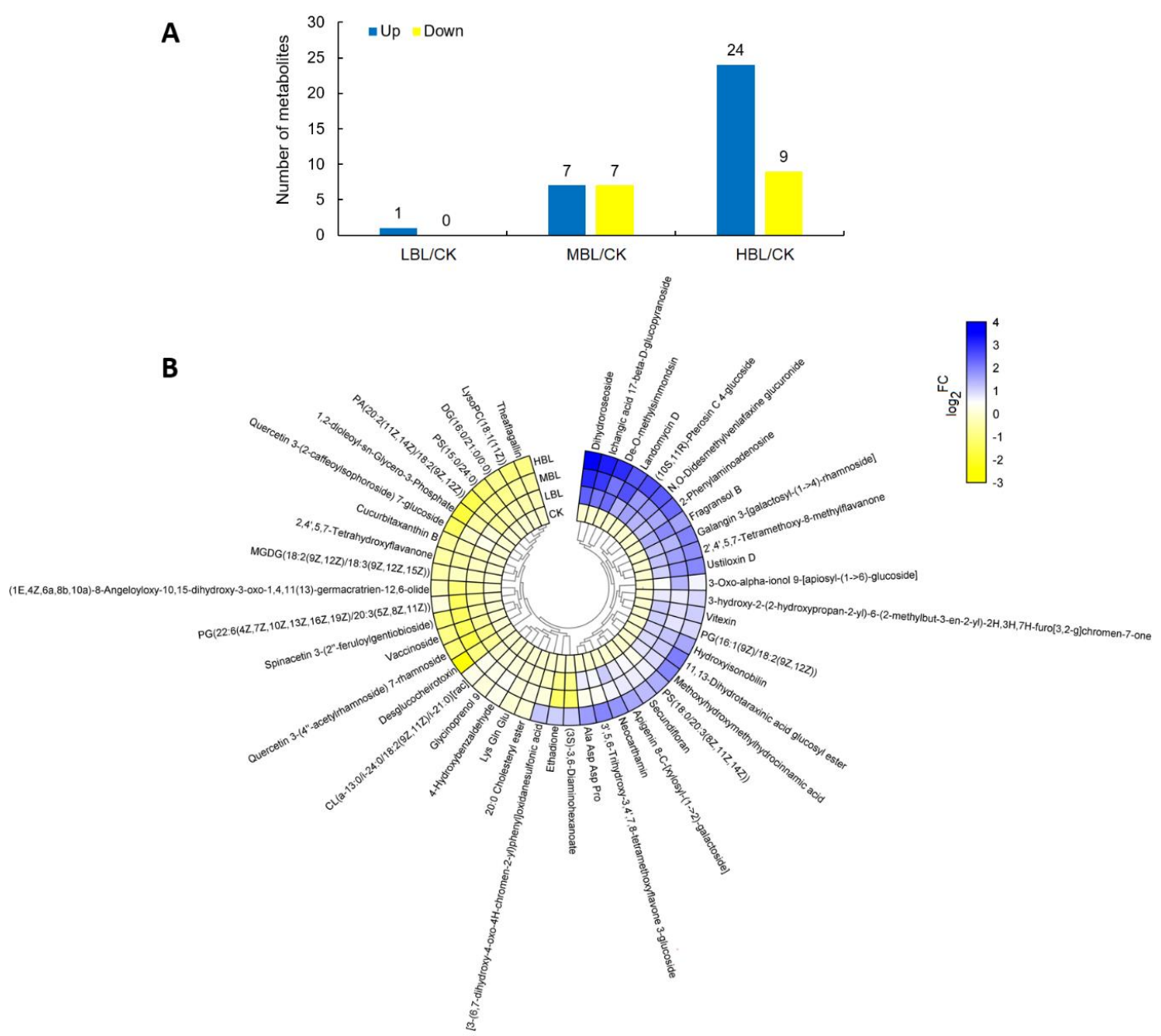

Figure 9. Statistics of the significantly changed metabolites (SCMs) between CK and low-intensity blue light (LBL), CK and medium-intensity blue light (MBL), and CK and HBL. (A) The number of SCMs in each comparison. (B) The SCM levels in response to different blue light intensities in tea plant shoots. The color bar represents the normalized fold change values.

\subsection{Coexpression Network Analysis}

To further identify modules related to flavonoid and lipid metabolism, the significantly changed flavonoids and lipids were combined with RNA-seq data to construct a coexpression network (Figure 10A). Seven modules (labeled in different colors) were identified in the dendrogram, where the gray module represents genes that were not assigned to specific modules. Remarkably, the red module showed a significant correlation with the accumulation pattern of flavonoids and lipids ( $\mathrm{r}>0.6$ or $\mathrm{r}<-0.6, p<0.05$ ) (Figure 10B and Table S4). Among these genes, 83 genes of the red module were negatively related to spinacetin 3- (2"- feruloylgentiobioside) and highly correlated with eleven lipids. Based on the eigengene connectivity (KME) values in the coexpression network, the top 50 node genes in the red module were selected to generate the coexpression subnetwork (Figure 10C). Among these genes, the hub gene CsMYB (TEA001045) had the highest KME value and was most strongly associated with other node genes. Additionally, the structural gene chalcone synthase (CsCHS, TEA018665) involved in flavonoid synthesis and the alcohol dehydrogenase (CSADH, TEA029314) gene involved in fatty acid degradation were located on the periphery of the network. The MYB TF family has been shown to play a vital role in plant flavonoid metabolism. These results indicate that this CsMYB member (TEA018665) may be involved in regulating flavonoids and lipid metabolism under different blue light intensities in tea plants. 

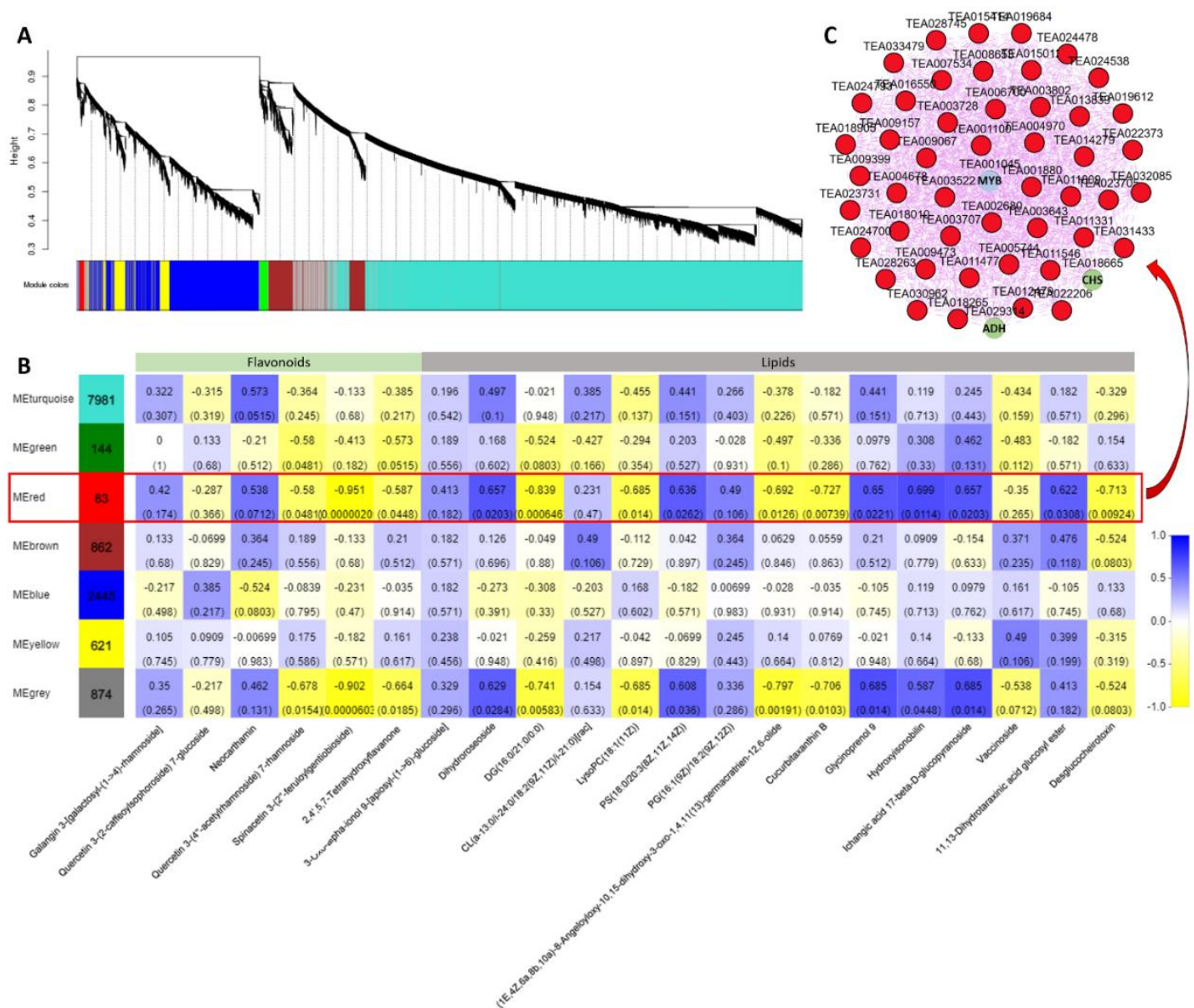

Figure 10. Coexpression network analysis. (A) Hierarchical cluster tree showing seven modules obtained by weighted gene co-expression network analysis (WGCNA). The gray modules represent genes that are not divided into specific modules. Each branch in the tree points to a gene. (B) Matrix of module-metabolite associations. The data of gene expression profiles under different blue light intensities and the change patterns of flavonoids and lipids in SCMs were combined to perform WGCNA analysis. The number of genes per module is shown in the left box. Correlation coefficients and $p$-values between modules and metabolites are shown at the row-column intersection. (C) Coexpression subnetwork analysis of red modules related to flavonoids and lipid accumulation. The top 50 nodes of the red module were selected to construct the network. The hub gene is shown in blue, and genes involved in flavonoid and lipid metabolism are shown in green.

\section{Discussion}

To date, many studies have focused on how different wavelengths of light affect several morphological processes in plants, including multiomic analysis on Arabidopsis [32], strawberry [33], lettuce [34] and tea plants [22]. Nonetheless, a comprehensive investigation of which metabolic pathways respond to different blue light intensities in plants, especially tea plants, has not been conducted. Thus, we aimed to elucidate the transcriptional and metabolic changes and key processes elicited in tea plant shoots in response to different intensities of blue LED light and help to elucidate which intensity of blue light can better regulate the growth and secondary metabolism of tea plants under cultivation conditions.

Previous studies have found that higher blue light intensity can significantly affect the accumulation of plant metabolites, such as carotenoids and lipids $[9,13]$. Our study clearly showed that the effect of supplementing $200 \mu \mathrm{mol} \mathrm{m}^{-2} \mathrm{~s}^{-1}$ blue LED light (HBL) on the growth and metabolism of tea plants was considerably stronger than that of supplementing $100 \mu \mathrm{mol} \mathrm{m}{ }^{-2} \mathrm{~s}^{-1}$ (MBL) and $50 \mu \mathrm{mol} \mathrm{m}{ }^{-2} \mathrm{~s}^{-1}$ (LBL): the number of differential genes and metabolites in tea plant shoots was significantly different 
under three blue LED light intensities (Figures 1C and 9A). These results imply that by appropriately increasing the intensity of blue light irradiation, the transcript reprogramming and metabolic flux redirection of tea plants can be improved to regulate the contents of tea plants.

Blue light is the major energy source for plant photosynthesis and can be recognized by photoreceptors that regulate plant development [35]. In cucumber leaves, blue light is essential to maintain the activity of photosystems II and I and improve the photosynthetic electron transfer ability [36]. Notably, our results showed that the 17 DEGs annotated to photosynthesis were significantly upregulated in HBL, especially five of them (four PsaB members from Photosystem I and one F-type ATPase- $\alpha$ ) that were upregulated more than 10-fold (Figure 5), which indicated the positive regulatory effect of high-intensity blue light on the energy metabolism of tea plants.

Lipids are one of the important subcellular components and play an essential role in plant development and signal transduction $[37,38]$. In addition, lipids greatly affect the flavor and aroma of brewed tea [39]. Therefore, research on the regulation of lipid biosynthesis in tea plants is of considerable interest. In this study, we found that 8 DEGs were enriched in the fatty acid metabolism pathway, although only FabG (TEA003420) was upregulated. Correspondingly, the largest number of differential metabolites belong to lipids and lipid-like molecules, including fatty acyls, glycerolipids, glycerophospholipids, prenol lipids, saccharolipids, steroids and steroid derivatives. These results indicate the stimulating effect of blue light on lipid biosynthesis, achieved by influencing related molecular pathways in tea plants. Similarly, Lakshmanan et al. [32] reported that Arabidopsis thaliana increased the flux of metabolic pathways, such as fatty acid biosynthesis, the Calvin cycle, the tricarboxylic acid cycle and glycolysis after blue light treatment, thereby enhancing the biosynthesis of lipids. Moreover, $200 \mu \mathrm{mol} \mathrm{m} \mathrm{m}^{-2} \mathrm{~s}^{-1}$ blue light can also regulate the maximum lipid content of Chlorella [13]. Interestingly, lipids are also closely related to plant photosynthesis. Lipids not only maintain the function of chloroplasts, which are the site of photosynthesis in higher plants but also directly participate in the mechanism of photosynthesis [40]. Our research revealed that high-intensity blue light can lead to the coexpression of genes in photosynthesis and lipid metabolism pathways and further regulate the synthesis of related metabolites.

Flavonoids are essential secondary metabolites in tea plants and are closely related to the quality of tea [41]. In addition, flavonoids have high antioxidant activity, which is potentially beneficial to human health [42]. Previous research has shown that whether blue light affects the synthesis of flavonoids depends on plant species [43]. Among these species, blue light can effectively improve the flavonoid production of arugula (Eruca saliva) but has no effect on bloody dock (Rumex sanguineus) and basil (Ocimunt basilicurn) [43]. In longan embryogenic calli, blue light selectively regulates the flux of flavonoid components, which inhibits the synthesis of rutin but promotes the accumulation of epicatechin [7]. Our results indicated that 19 structural genes involved in flavonoid synthesis were markedly downregulated in HBL. However, these genes have no significant changes in LBL and MBL, indicating that high-intensity blue light completely inhibits the molecular pathway of flavonoid synthesis. Meanwhile, we found that the most abundant TF families in DEGs were bHLH and MYB, which have been shown to be widely involved in the regulation of plant flavonoids $[44,45]$. We also observed that most bHLH (10/11) and MYB (6/8) TFs were coordinately downregulated in HBL with structural DEGs for flavonoid synthesis. Previous studies have elucidated a consistent regulatory mechanism in tea plants. For instance, CsbHLH (TEA003964) acts as a potential repressor to negatively regulate the expression of downstream flavonoid synthesis genes in tea plants [46]. CsMYB5a and CsMYB5e were also demonstrated to be involved in the biosynthesis regulation of multiple flavonoid components [47]. Based on WGCNA analysis, we further identified that CsMYB (TEA001045) may be a hub gene involved in the negative regulation of flavonoid and lipid metabolism by blue light (Figure 10), possibly by affecting structural node genes, such as CsCHS (TEA018665) and CsADH (TEA029314). 


\section{Materials and Methods}

\subsection{Tea Plant Materials and Blue Light Treatments}

One-year-old potted tea plants (Camellia sinensis cv. 'Fujian Shuixian') (Approved by the China Crop Variety Approval Committee in 1985, No. GS 13009-1985) cultivated in the Fujian Agriculture and Forestry University tea plant germplasm collection garden (Fuzhou, China) were selected as the materials. The tea plants were transferred to four controlled incubators for 30 days with a white LED light at an intensity of $100 \mu \mathrm{mol} \mathrm{m} \mathrm{s}^{-1}$ during the $12-\mathrm{h}$ photoperiod. The temperature and humidity were controlled at $25 \pm 3{ }^{\circ} \mathrm{C}$ and $75 \pm 3 \%$, respectively. Subsequently, as shown in Figure 11, the tea plants in the other three incubators were exposed to white light during the daytime and provided three complementary blue LED lights $(450 \mathrm{~nm})$ of different light intensities, including $50 \mu \mathrm{mol} \mathrm{m}^{-2} \mathrm{~s}^{-1}$ (LBL), $100 \mu \mathrm{mol} \mathrm{m} \mathrm{m}^{-2} \mathrm{~s}^{-1}$ (MBL) and $200 \mu \mathrm{mol} \mathrm{m} \mathrm{m}^{-2}$ (HBL). After 14 days, the young shoots (one bud and two leaves) of each treatment were collected for transcriptome and metabolome analyses, and three biological replicates were performed.

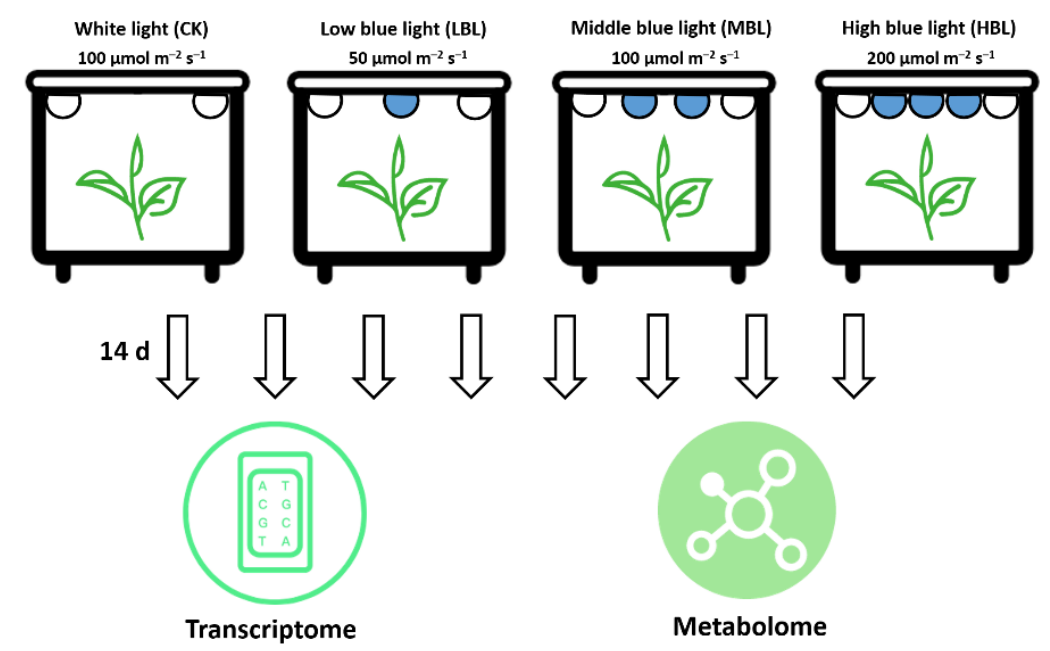

Figure 11. Schematic illustration of the treatment and omics analyses of tea plants under different blue LED light intensities.

\subsection{RNA-Seq Processing and Data Analysis}

Total RNA was extracted from the samples using an RNAprep Pure Plant Kit (DP441, TIANGEN, Beijing, China) according the manufacturer's instructions, and genomic DNA was removed using DNase I. The high-quality RNA samples were used to construct a sequencing library and sequenced using an Illumina Novaseq 6000 ( $2 \times 150$-bp read length). The raw paired end reads were trimmed and quality-controlled by SeqPrep (https://github.com/jstjohn/SeqPrep) and Sickle (https://github. com/najoshi/sickle) with default parameters. Then, clean reads were separately aligned to the tea plant genome [16] using TopHat 2.1.1 [48]. The expression level of each transcript was calculated according to the transcripts per million reads (TPM) method. RSEM (http://deweylab.biostat.wisc.edu/ rsem/) [49] and EdgeR package (http://www.bioconductor.org/packages/2.12/bioc/html/edgeR.html) [50] were utilized for quantitative gene abundance and differential expression analysis, respectively. Genes with FDR $\leq 0.05$ and fold change $\geq 2$ were considered to be differentially expressed genes (DEGs). Furthermore, iPath3.0 (http://pathways.embl.de) [31] was used to visualize and analyze the metabolic pathways of DEGs. Gene Ontology (GO) and Kyoto Encyclopedia of Genes and Genomes (KEGG) enrichment analyses were performed by Goatools (https://github.com/tanghaibao/Goatools) and KOBAS 2.1.1 (http://kobas.cbi.pku.edu.cn/download.php) [51]. To visualize the transcriptional abundance of DEGs, heatmaps were generated using TBtools [52], and chord plots were generated using the free online platform of Majorbio Cloud Platform (www.majorbio.com). 


\subsection{Gene Expression Analysis by Quantitative Real-time PCR ( $q$ RT-PCR)}

cDNA synthesis and qRT-PCR tests were performed to verify the reliability of the RNA-Seq data according to previous methods [53]. CsGAPDH (GE651107) was used as a reference control, and the primers of validated genes were designed using Primer3Plus (http://www.bioinformatics.nl/cgi-bin/ primer3plus/primer3plus.cgi). The primer information is listed in Table S5. All samples were analyzed in three biological replicates. The relative expression level was calculated using the $2^{-\Delta \Delta \mathrm{Ct}}$ method [54].

\subsection{Metabolite Profiling Analysis}

The 100-mg tea plant samples were accurately weighed, and the metabolites were extracted using a $400 \mu \mathrm{L}$ methanol:water $(4: 1, v / v)$ solution. The mixture was settled at $-20^{\circ} \mathrm{C}$ and processed by a high-throughput tissue crusher Wonbio-96c (Wanbo Biotechnology, Shanghai, China) at $50 \mathrm{~Hz}$ for $6 \mathrm{~min}$, then vortexed for $30 \mathrm{~s}$ and ultrasonicated at $5^{\circ} \mathrm{C}$ for $30 \mathrm{~min}$. The samples were placed at $-20^{\circ} \mathrm{C}$ for $30 \mathrm{~min}$ to precipitate proteins. After centrifugation at $13,000 \mathrm{rpm}$ for $15 \mathrm{~min}$ at $4{ }^{\circ} \mathrm{C}$, the supernatant was carefully transferred to sample vials. The quality control $(\mathrm{QC})$ samples were prepared by mixing equal volumes of all samples to monitor the accuracy and stability of the method.

Metabolites were profiled using a UPLC-Triple-TOF-MS-based platform, and chromatographic separation was performed on an ExionLC ${ }^{\mathrm{TM}}$ AD system (AB Sciex, Los Angeles, CA, USA) equipped with an ACQUITY UPLC BEH C18 column $(1.7 \mu \mathrm{m}, 100 \mathrm{~mm} \times 2.1 \mathrm{~mm}$, Waters, Milford, MA, USA). Phase A was water with $0.1 \%$ formic acid $(v / v)$, and phase B was $0.1 \%$ formic acid in acetonitrile: isopropanol $(1: 1, v / v)$. The sample injection volume was $20 \mu \mathrm{L}$, and the flow rate was set to $0.4 \mathrm{~mL} / \mathrm{min}$ with a column temperature of $40{ }^{\circ} \mathrm{C}$. The solvent gradient was as follows: from 0 to $3 \mathrm{~min}, 95 \% \mathrm{~A}$ : $5 \%$ B to $80 \%$ A: $20 \%$ B; from 3 to $9 \mathrm{~min}, 80 \%$ A: $0 \%$ B to $5 \%$ A: $95 \%$ B; from 9 to $13 \mathrm{~min}, 5 \%$ A: $95 \%$ B to $5 \% \mathrm{~A}: 95 \% \mathrm{~B}$; from 13 to $13.1 \mathrm{~min}, 5 \% \mathrm{~A}$ : $95 \% \mathrm{~B}$ to $95 \% \mathrm{~A}: 5 \% \mathrm{~B}$, from 13.1 to $16 \mathrm{~min}, 95 \% \mathrm{~A}: 5 \%$ $\mathrm{B}$ to $95 \%$ A: $5 \%$ B. MS analysis was performed using a quadrupole-time-of-flight mass spectrometer (Triple $\mathrm{TOF}^{\mathrm{TM}} 5600+$, AB Sciex, Los Angeles, CA, USA) equipped with an electrospray ionization (ESI) source operating in positive mode and negative mode. The optimal parameters were set as follows: source temperature, $500{ }^{\circ} \mathrm{C}$; curtain gas, $30 \mathrm{psi}$; both ion sources GS1 and GS2, 50 psi; ion-spray voltage floating, $-4000 \mathrm{~V}$ in negative mode and $5000 \mathrm{~V}$ in positive mode; declustering potential (DP), $80 \mathrm{~V}$; collision energy (CE), 20-60 V rolling for MS/MS. Data acquisition was performed with the data-dependent acquisition (DDA) mode with an $\mathrm{m} / \mathrm{z}$ range between $50-1000$.

Raw data were imported into Progenesis QI 2.3 (Nonlinear Dynamics, Waters, MA, USA) for peak detection and alignment. The preprocessing results contained the $\mathrm{m} / \mathrm{z}$ values, RT, and peak intensity. Mass spectra of these metabolic features were identified by using accurate mass, MS/MS fragment spectra and isotope ratio differences with searches in internal databases and public databases. The variable importance of the projection (VIP) score generated from orthogonal partial least squares discriminate analysis (OPLS-DA) was used to determine the best differentiated metabolites between CK and treatments. Metabolites with VIP $\geq 1.0$ and $p$-value $\leq 0.05$ were defined as significantly changed metabolites (SCMs). Multivariate statistical analysis was performed using R package ropls version 1.6.2 (http://bioconductor.org/packages/release/bioc/html/ropls.html).

\subsection{Coexpression Analysis}

The gene coexpression network was constructed using the R package WGCNA [55] to identify modules of highly correlated genes and metabolites based on the filtering data (mean expression level $\geq 1$, coefficient of variation $\geq 0.1$ ). After filtering, the abundance of 13,010 genes and 22 metabolites was used to build a signed coexpression network by calculating Pearson's correlations. The soft-thresholding power of the correlation network was set at 9 , and the minimum module size was equal to 30. The core coexpression modules were visualized using Cytoscape 3.4.0 [56]. 


\section{Conclusions}

Our study compared the effects of three levels of blue light intensity on the transcripts and metabolites of tea plants and found that high-intensity blue light (HBL) can significantly affect the secondary metabolism of tea plants. By mining the transcriptome data, it was found that the blue light-responsive genes were related to photosynthesis, lipid metabolism and flavonoid synthesis, which is consistent with the annotation of the significantly changed metabolites. In addition, based on the annotation of transcription factors and WGCNA analysis, we further identified that CsMYB (TEA001045) may be the hub gene regulating the effects of blue light on lipid and flavonoid metabolism. These results may provide a reference for future research investigating the regulation of woody plants by blue light.

Supplementary Materials: The following can be found at http://www.mdpi.com/1422-0067/21/13/4606/s1. Table S1. The top 10 GO terms and enriched DEGs between CK and HBL. Table S2. The top 10 KEGG pathways and enriched DEGs between CK and HBL. Table S3. Significantly changed metabolites in different treatments. Table S4. The genes and their module from WGCNA. Table S5. The primers for qRT-PCR.

Author Contributions: N.Y., F.Z., J.Y. and P.W. conceived and designed the project; P.W., S.C. and X.C. (Xuejin Chen) performed the experiments; P.W., M.G. and X.C. (Xiaomin Chen) analyzed the data; P.W. wrote the paper. All authors reviewed and approved the final manuscript.

Funding: This research received no external funding.

Acknowledgments: This research was funded by the Fujian Province "2011 Collaborative Innovation Center", Chinese Oolong Tea Industry Innovation Center special project (J2015-75), the Fujian Agriculture and Forestry University Project for Technological Innovation of Tea Industry Chain and Service System Construction: 2020-01, the Fujian Agriculture and Forestry University Science and Technology Innovation Special Fund (CXZX2017181), and the Scientific Research Foundation of Graduate School of Fujian Agriculture and Forestry University (324-1122yb060).

Conflicts of Interest: The authors declare no conflict of interest.

\section{Abbreviations}

$\begin{array}{ll}\text { TF } & \text { Transcription factor } \\ \text { LEDs } & \text { Light emitting diodes } \\ \text { TPM } & \text { Transcripts Per Million reads } \\ \text { DEGs } & \text { Differentially expressed genes } \\ \text { GO } & \text { Gene Ontology } \\ \text { KEGG } & \text { Kyoto Encyclopedia of Genes and Genomes } \\ \text { WGCNA } & \text { Weighted gene co-expression network analysis } \\ \text { QC } & \text { Quality control } \\ \text { ESI } & \text { Electrospray ionization } \\ \text { DP } & \text { De-clustering potential } \\ \text { CE } & \text { Collision energy } \\ \text { DDA } & \text { Data dependent acquisition } \\ \text { VIP } & \text { Variable importance of the projection } \\ \text { OPLS-DA } & \text { orthogonal partial least squares discriminate analysis } \\ \text { SCMs } & \text { Significantly changed metabolites } \\ \text { CHS } & \text { chalcone synthase } \\ \text { ADH } & \text { alcohol dehydrogenase }\end{array}$

\section{References}

1. Chory, J. Light signal transduction: An infinite spectrum of possibilities. Plant J. 2010, 61, 982-991. [CrossRef] [PubMed]

2. Christie, M.J. Phototropin blue-light receptors. Annu. Rev. Plant Biol. 2007, 58, 21-45. [CrossRef] [PubMed]

3. Liscum, E.; Briggs, W.R. Mutations in the NPH1 locus of Arabidopsis disrupt the perception of phototropic stimuli. Plant Cell 1995, 7, 473-485. 
4. Kinoshita, T.; Doi, M.; Suetsugu, N.; Kagawa, T.; Wada, M.; Shimazaki, K. Phot1 and phot2 mediate blue light regulation of stomatal opening. Nature 2001, 414, 656-660. [CrossRef] [PubMed]

5. Cashmore, R.A. Cryptochromes: Blue light receptors for plants and animals. Science 1999, 284, 760-765. [CrossRef] [PubMed]

6. Terfa, M.T.; Solhaug, K.A.; Gislerød, H.R.; Olsen, J.E.; Torre, S. A high proportion of blue light increases the photosynthesis capacity and leaf formation rate of Rosa $\times$ hybrida but does not affect time to flower opening. Physiol. Plant. 2013, 148, 146-159. [CrossRef]

7. Li, H.; Lin, Y.; Chen, X.; Bai, Y.; Wang, C.; Xu, X.; Wang, Y.; Lai, Z. Effects of blue light on flavonoid accumulation linked to the expression of miR393, miR394 and miR395 in longan embryogenic calli. PLoS ONE 2018, 13, e191444. [CrossRef]

8. $\quad$ Li, H.; Lyu, Y.; Chen, X.; Wang, C.; Yao, D.; Ni, S.; Lin, Y.; Chen, Y.; Zhang, Z.; Lai, Z. Exploration of the effect of blue light on functional metabolite accumulation in longan embryonic calli via RNA sequencing. Int. J. Mol. Sci. 2019, 20, 441. [CrossRef]

9. Zhang, L.; Ma, G.; Yamawaki, K.; Ikoma, Y.; Matsumoto, H.; Yoshioka, T.; Ohta, S.; Kato, M. Effect of blue LED light intensity on carotenoid accumulation in citrus juice sacs. J. Plant Physiol. 2015, 188, 58-63. [CrossRef]

10. Yuan, Z.; Deng, L.; Yin, B.; Yao, S.; Zeng, K. Effects of blue LED light irradiation on pigment metabolism of ethephon-degreened mandarin fruit. Postharvest Biol. Tec. 2017, 134, 45-54. [CrossRef]

11. Deng, L.; Yuan, Z.; Xie, J.; Yao, S.; Zeng, K. Sensitivity to ethephon degreening treatment is altered by blue LED light irradiation in mandarin fruit. J. Agric. Food Chem. 2017, 65, 6158-6168. [CrossRef] [PubMed]

12. Jung, B.K.; Kim, C.S.; Jung, J. Blue light effect on the fatty acid composition of membrane lipid of plant leaves. Korean J. Environ. Agric. 1992, 11, 261-268.

13. Atta, M.; Idris, A.; Bukhari, A.; Wahidin, S. Intensity of blue LED light: A potential stimulus for biomass and lipid content in fresh water microalgae Chlorella vulgaris. Bioresour. Technol. 2013, 148, 373-378. [CrossRef]

14. Wang, P.; Zheng, Y.; Guo, Y.; Liu, B.; Jin, S.; Liu, S.; Zhao, F.; Chen, X.; Sun, Y.; Yang, J.; et al. Widely targeted metabolomic and transcriptomic analyses of a novel albino tea mutant of 'Rougui'. Forests 2020, 11, 229. [CrossRef]

15. Xia, E.H.; Zhang, H.B.; Sheng, J.; Li, K.; Zhang, Q.J.; Kim, C.; Zhang, Y.; Liu, Y.; Zhu, T. The tea tree genome provides insights into tea flavor and independent evolution of caffeine biosynthesis. Mol. Plant 2017, 10, 866-877. [CrossRef]

16. Wei, C.; Yang, H.; Wang, S.; Zhao, J.; Liu, C.; Gao, L.; Xia, E.; Lu, Y.; Tai, Y.; She, G. Draft genome sequence of Camellia sinensis var. sinensis provides insights into the evolution of the tea genome and tea quality. Proc. Natl. Acad. Sci. USA 2018, 115, E4151-E4158. [CrossRef]

17. Yang, Z.; Kobayashi, E.; Katsuno, T.; Asanuma, T.; Fujimori, T.; Ishikawa, T.; Tomomura, M.; Mochizuki, K.; Watase, T.; Nakamura, Y.; et al. Characterisation of volatile and non-volatile metabolites in etiolated leaves of tea (Camellia sinensis) plants in the dark. Food Chem. 2012, 135, 2268-2276. [CrossRef] [PubMed]

18. Zhang, Q.; Shi, Y.; Ma, L.; Yi, X.; Ruan, J. Metabolomic analysis using ultra-performance liquid chromatography-quadrupole-time of flight mass spectrometry (UPLC-Q-TOF MS) uncovers the effects of light intensity and temperature under shading treatments on the metabolites in tea. PLoS ONE 2014, 9, e112572. [CrossRef] [PubMed]

19. Zhang, Q.; Liu, M.; Ruan, J. Metabolomics analysis reveals the metabolic and functional roles of flavonoids in light-sensitive tea leaves. BMC Plant Biol. 2017, 17, 64. [CrossRef]

20. Liu, L.; Li, Y.; She, G.; Zhang, X.; Jordan, B.; Chen, Q.; Zhao, J.; Wan, X. Metabolite profiling and transcriptomic analyses reveal an essential role of UVR8-mediated signal transduction pathway in regulating flavonoid biosynthesis in tea plants (Camellia sinensis) in response to shading. BMC Plant Biol. 2018, 18, 233. [CrossRef]

21. Fu, X.; Chen, Y.; Mei, X.; Katsuno, T.; Kobayashi, E.; Dong, F.; Watanabe, N.; Yang, Z. Regulation of formation of volatile compounds of tea (Camellia sinensis) leaves by single light wavelength. Sci. Rep. 2015, 5, 16858. [CrossRef]

22. Zheng, C.; Ma, J.; Ma, C.; Shen, S.; Liu, Y.; Chen, L. Regulation of growth and flavonoid formation of tea plants (Camellia sinensis) by blue and green light. J. Agric. Food Chem. 2019, 67, 2408-2419. [CrossRef] [PubMed]

23. Fankhauser, C.; Chory, J. Light control of plant development. Annu. Rev. Cell Dev. Biol. 1997, 13, $203-229$. [CrossRef] 
24. Ouzounis, T.; Rosenqvist, E.; Ottosen, C. Spectral effects of artificial light on plant physiology and secondary metabolism: A review. Hortscience 2015, 50, 1128-1135. [CrossRef]

25. Bantis, F.; Smirnakou, S.; Ouzounis, T.; Koukounaras, A.; Radoglou, K. Current status and recent achievements in the field of horticulture with the use of light-emitting diodes (LEDs). Sci. Hortic. 2018, 235, 437-451. [CrossRef]

26. Hernández, R.; Kubota, C. Physiological responses of cucumber seedlings under different blue and red photon flux ratios using LEDs. Env. Exp. Bot. 2015, 29, 66-74. [CrossRef]

27. Brown, C.S.; Schuerger, A.C.; Sager, J.C. Growth and photomorphogenesis of pepper plants under red light-emitting diodes with supplemental blue or far-red lighting. J. Am. Soc. Hortic. Sci. 1995, 120, 808-813. [CrossRef] [PubMed]

28. Nhut, D.T.; Hong, L.T.A.; Watanabe, H.; Goi, M.; Tanaka, M. Growth of banana plantlets cultured in vitro under red and blue light-emitting diode (LED) irradiation source. Acta. Hortic. 2002, 575, 117-124. [CrossRef]

29. Li, Y.; Xin, G.; Wei, M.; Shi, Q.; Yang, F.; Wang, X. Carbohydrate accumulation and sucrose metabolism responses in tomato seedling leaves when subjected to different light qualities. Sci. Hortic. 2017, 225, 490-497. [CrossRef]

30. Poudel, P.R.; Kataoka, I.; Mochioka, R. Effect of red- and blue-light-emitting diodes on growth and morphogenesis of grapes. Plant Cell Tiss. Org. 2008, 92, 147-153. [CrossRef]

31. Darzi, Y.; Letunic, I.; Bork, P.; Yamada, T. iPath3.0: Interactive pathways explorer v3. Nucleic Acids. Res. 2018, 46, 510-513. [CrossRef]

32. Lakshmanan, M.; Lim, S.H.; Mohanty, B.; Kim, J.K.; Lee, D.L. Unraveling the light-specific metabolic and regulatory signatures of rice through combined in silico modeling and multiomics analysis. Plant Physiol. 2015, 169, 3002-3020. [PubMed]

33. Zhang, Y.; Jiang, L.Y.; Li, Y.L.; Chen, Q.; Ye, Y.T.; Zhang, Y.; Luo, Y.; Sun, B.; Wang, X.R.; Tang, H. Effect of red and blue light on anthocyanin accumulation and differential gene expression in strawberry (Fragaria $\times$ ananassa). Molecules 2018, 23, 820. [CrossRef] [PubMed]

34. Kitazaki, K.; Fukushima, A.; Nakabayashi, R.; Okazaki, Y.; Kobayashi, M.; Mori, T.; Nishizawa, T.; Reyes-Chin-Wo, S.; Michelmore, R.W.; Saito, K.; et al. Metabolic reprogramming in leaf lettuce grown under different light quality and intensity conditions using narrow-band LEDs. Sci. Rep. 2018, 8, 7914. [CrossRef] [PubMed]

35. Briggs, W.R. Phototropins 1 and 2: Versatile plant blue-light receptors. Trends Plant Sci. 2002, 7, $204-210$. [CrossRef]

36. Miao, Y.; Wang, X.; Gao, L.; Chen, Q.; Qu, M. Blue light is more essential than red light for maintaining the activities of photosystem II and I and photosynthetic electron transport capacity in cucumber leaves. J. Integr. Agr. 2016, 15, 87-100. [CrossRef]

37. Leeuwen, W.V.; Krész, L.; B Gre, L.; Munnik, T. Learning the lipid language of plant signalling. Trends Plant Sci. 2004, 9, 378-384. [CrossRef] [PubMed]

38. Kobayashi, K.; Endo, K.; Wada, H. Roles of lipids in photosynthesis. Subcell Biochem. 2016, 86, 21-49. [PubMed]

39. Ho, C.T.; Zheng, X.; Li, S. Tea aroma formation. Food Sci. Hum. Wellness 2015, 4, 9-27. [CrossRef]

40. Jouhet, J.; Dubots, E.; Maréchal, E.; Block, M.A. Lipids in photosynthesis: Lipid trafficking in plant photosynthetic cells. Adv. Photosynth. Respir. 2009, 30, 349-372.

41. Sultana, T.; Stecher, G.; Mayer, R.; Trojer, L.; Qureshi, M.N.; Abel, G.; Popp, M.; Bonn, G.K. Quality assessment and quantitative analysis of flavonoids from tea samples of different origins by HPLC-DAD-ESI-MS. J. Agric. Food Chem. 2008, 56, 3444-3453. [CrossRef] [PubMed]

42. Knekt, P.; Kumpulainen, J.; Järvinen, R.; Rissanen, H.; Heli Vaara, M.; Reunanen, A.; Hakulinen, T.; Aromaa, A. Flavonoid intake and risk of chronic diseases. Am. J. Clin. Nutr. 2002, 76, 560-568. [CrossRef]

43. Taulavuori, K.; Pyysalo, A.; Taulavuori, E.; Julkunen-Tiitto, R. Responses of phenolic acid and flavonoid synthesis to blue and blue-violet light depends on plant species. Env. Exp. Bot. 2018, 150, 183-187. [CrossRef]

44. Dubos, C.; Stracke, R.; Grotewold, E.; Weisshaar, B.; Martin, C.; Lepiniec, L.C. MYB transcription factors in Arabidopsis. Trends Plant Sci. 2010, 15, 573-581. [CrossRef] [PubMed]

45. Heim, M.A.; Marc, J.; Martin, W.; Cathie, M.; Bernd, W.; Bailey, P.C. The basic helix-loop-helix transcription factor family in plants: A genome-wide study of protein structure and functional diversity. Mol. Biol. Evol. 2003, 20, 735-747. [CrossRef] 
46. Zhu, J.; Xu, Q.; Zhao, S.; Xia, X.; Yan, X.; An, Y.; Mi, X.; Guo, L.; Samarina, L.; Wei, C. Comprehensive co-expression analysis provides novel insights into temporal variation of flavonoids in fresh leaves of the tea plant (Camellia sinensis). Plant Sci. 2020, 290, 110306. [CrossRef]

47. Jiang, X.; Huang, K.; Zheng, G.; Hou, H.; Wang, P.; Jiang, H.; Zhao, X.; Li, M.; Zhang, S.; Liu, Y.; et al. CsMYB5a and CsMYB5e from Camellia sinensis differentially regulate anthocyanin and proanthocyanidin biosynthesis. Plant Sci. 2018, 270, 209-220. [CrossRef]

48. Trapnell, C.; Roberts, A.; Goff, L.; Pertea, G.; Kim, D.; Kelley, D.R.; Pimentel, H.; Salzberg, S.L.; Rinn, J.L.; Pachter, L. Differential gene and transcript expression analysis of RNA-seq experiments with TopHat and Cufflinks. Nat. Protoc. 2014, 7, 562-578. [CrossRef]

49. Dewey, C.N.; Li, B. RSEM: Accurate transcript quantification from RNA-Seq data with or without a reference genome. BMC Bioinform. 2011, 12, 323.

50. Robinson, M.D.; McCarthy, D.J.; Smyth, G.K. edgeR: A Bioconductor package for differential expression analysis of digital gene expression data. Bioinform. 2010, 26, 139-140. [CrossRef]

51. Xie, C.; Mao, X.; Huang, J.; Ding, Y.; Wu, J.; Dong, S.; Kong, L.; Gao, G.; Li, C.Y.; Wei, L. KOBAS 2.0: A web server for annotation and identification of enriched pathways and diseases. Nucleic Acids. Res. 2011, 39, 316-322. [CrossRef]

52. Chen, C.J.; Chen, H.; Zhang, Y.; Thomas, H.R.; Frank, M.H.; He, Y.; Xia, R. TBtools-An integrative toolkit developed for interactive analyses of big biological data. Mol. Plant. 2020. [CrossRef] [PubMed]

53. Wang, P.J.; Zheng, Y.C.; Guo, Y.C.; Chen, X.J.; Sun, Y.; Yang, J.F.; Ye, N.X. Identification, expression, and putative target gene analysis of nuclear factor-Y (NF-Y) transcription factors in tea plant (Camellia sinensis). Planta 2019, 250, 1671-1686. [CrossRef] [PubMed]

54. Livak, K.J.; Schmittgen, T.D. Analysis of relative gene expression data using real-time quantitative PCR and the 2(-Delta Delta C(T)) Method. Methods 2001, 25, 402-408. [CrossRef] [PubMed]

55. Langfelder, P.; Horvath, S. WGCNA: An R package for weighted correlation network analysis. BMC Bioinform. 2008, 9, 559. [CrossRef]

56. Kohl, M.; Wiese, S.; Warscheid, B. Cytoscape: Software for visualization and analysis of biological networks. Methods Mol. Biol. 2011, 696, 291-303. 